\title{
Another graph partitioning optimization problem
}

\author{
Thinh D. Nguyen
}

\begin{abstract}
This paper defines yet another graph partitioning problem in the form of a combinatorial optimization problem. A reduction from 3-WAY CUT (cf. [1]) problem shows that our claim holds.
\end{abstract}

Keywords. cut, graph, multiway, optimization, partition

\section{Introduction and notations}

We start from 3-WAY CUT problem, which is $N P$-hard even when all edge weights equal to 1 (cf. [1]). For clarity, we give the description of that problem here.

\section{Definition 1. (Problem 3-WAY CUT)}

Input: a graph $G=(V, E)$ and terminals $s_{1}, s_{2}, s_{3} \in V$

Output: a minimum edge set $E^{\prime} \subset E$ such that the removal of $E^{\prime}$ from $E$ disconnects each terminal $s_{i}$ from the others

The decision version is to find out if it is possible to disconnect $s_{1}, s_{2}, s_{3}$ by removing no more than e edges, where e is part of the input.

Above is the definition of the problem from which we will reduce to our problem, which is defined as follows. We denote our problem by GRAPH Partition SumSquared.

\section{Definition 2. (Problem Graph Partition SumSquared)}

Input: An integer $e$, and a connected graph $G=(V, E)$ together with a vertex weight function $w: V \rightarrow \mathbb{N}$

Output: A partition of $G$, namely $G_{p}=\left(V, E_{p}\right)$, obtained by removing e edges from $E$ which maximizes:

$$
\max \sum_{G_{i} \in\left\{G_{1}, G_{2}, \ldots, G_{k}\right\}} \frac{1}{\left|G_{i}\right|} \sum_{v_{j} \in V_{i}} w\left(v_{j}\right)^{2}
$$

where $G_{p}=G_{1} \cup G_{2} \cup \ldots \cup G_{k}$ and elements of $G_{p}$ are disjoint. And, $V_{i}$ is the vertex set of $G_{i}$.

As before, the decision version of the problem is to find out if there exists a partition which objective value is at least some value $L$, where $L$ is part of the input. 
Some explanation in plain words are given as to improve the naturalness of the above definition. So, in Graph Partition SumSquared, we wish to partition a graph by removing $e$ edges to maximize an objective function. The objective function computes for each of the resulting disjoint subgraphs the sum of its vertex weight, squares the obtained value, and divides by the number of vertices, and finally sum over all the disjoint subgraphs.

In the next section, we prove the following claim.

Claim 3. 3-way Cut $\leq_{p}$ Graph Partition SumSquared

\section{Description of the construction}

As mentioned in the abstract, we reduce from 3-WAY CUT. And in this section, we describe the construction and proof of correctness of the details in our reduction.

Proof. An instance of the decision problem 3-WAY CuT includes a graph $G=(V, E)$ with $|V|=n$, three terminals $s_{1}, s_{2}, s_{3}$, and an integer $e$. It is a YES instance if there exists a set $E^{\prime} \subset E$ consisting of $e$ edges, the removal of which would disconnect the three terminals from each other. Otherwise, the given instance is a No instance. From this instance, we would like to construct an instance of Graph Partition SumSquared. We keep all the original graph $G$ as is, and add some new vertices to it. For each terminal $s_{i}$, we add $N$ more new vertices adjacent to it, where $N$ is a large enough natural number. The exact magnitude is clear from what follows. Denote our constructed graph by $G^{\prime}$.

All the vertices of $G^{\prime}$ are weighted 0 , except for $s_{1}, s_{2}, s_{3}$, whose weight are $1,10,100$, respectively. Denote the extended weight function by $w^{\prime}$. It is immediate from the description here that $(*)$ the three terminals can be disconnected from each other by removing $e$ edges in $G$, if and only if they can also be disconnected from each other by removing $e$ edges in $G^{\prime}$.

If every partition $G_{p}^{\prime}$ of $G^{\prime}$ disconnecting $s_{1}, s_{2}, s_{3}$ must have an objective value no less than $\frac{10101}{N+n}$. This is because: (i) every subgraph without any terminal contributes 0 to the objective value, (ii) each other partition, containing exactly one terminal $s_{i}$, cannot have more than $N+n$ vertices, so its contribution to the objective value would be no less than $\frac{w\left(s_{i}\right)}{N+n}$, (iii) summing over all the subgraphs of the partition $G_{p}^{\prime}$, we obtain the claimed lower bound.

On the other hand, every partition $G_{p}^{\prime}$ of $G$, fails to disconnect $s_{1}, s_{2}, s_{3}$ must have an objective value no more than $\frac{10060.5}{N-e}$. It is worth noting that 
after removing $e$ edges, if a subgraph contains exactly $j$ terminals, then it has no less than $j N-e$ vertices; observe that before the removal, it has at least $j N$ vertices, and the removal removes at most $e$ vertices from it. Now, to see why the upper bound $\frac{10060.5}{N-e}$ holds, we consider two cases. In the first case, $s_{3}$ and one of $s_{1}$ or $s_{2}$ are in the same subgraph. In this case, the subgraph, containing two terminals including $s_{3}$, would contribute at most $\frac{10100}{2 N-e}$ to the objective value. The other subgraph, containing the remaining terminal, would contribute at most $\frac{100}{N-e}$ to the objective value. Therefore the total is at most $\frac{10200}{2 N-e}$. In the second case, $s_{3}$ is in one subgraph and $s_{1}, s_{2}$ are in one other subgraph. A similar computation shows that in this case the total is at most $\frac{10000}{N-e}+\frac{121}{2 N-e}$. So, for large enough value of $N$, the common lower bound would be taken as $\frac{10060.5}{N-e}$ as claimed.

Having established a lower bound for a feasible partition and an upper bound for an infeasible partition, we observe that $\frac{10101}{N+n}>\frac{10060.5}{N-e}$ for large enough $N$. Combining this with $(*)$, we can conclude that $G$ can be 3 -way partitioned by removing $e$ edges, if and only if $G^{\prime}$ has a partition removing $e$ edges, the objective value of which is at least $\frac{10101}{N+n}$. Set $L=\frac{10101}{N+n}$, we obtain, as a result of this reduction, an instance $\left(G^{\prime}, w^{\prime}, e, L\right)$ of GRAPH PARTition SumSquared.

\section{References}

[1] E. Dahlhaus, D. S. Johnson, C. H. Papadimitriou, P. D. Seymour, M. Yannakakis The Complexity of Multiterminal Cuts 